\title{
Non-Destructive Analysis of Explanted Coronary Artery Stents
}

\author{
Dóra Károly ${ }^{1}$, Lilla Asztalos ${ }^{1}$, Tamás Micsík ${ }^{2}$, Péter János \\ Szabó ${ }^{1}$
}

${ }^{1}$ Department of Materials Science and Engineering, Faculty of Mechanical Engineering, Budapest University of Technology and Economics; Bertalan L. u. 7, H-1111 Budapest, Hungary; kdora@eik.bme.hu, lilla@eik.bme.hu, szpj@eik.bme.hu

${ }^{2}$ I. Department of Pathology and Experimental Cancer Research, Semmelweis University; Üllői út 26, H-1085 Budapest, Hungary; micsik.tamas@med.semmelweis-univ.hu

Abstract: Endovascular stents are used to effectively treat atherosclerosis during angioplasty. Nowadays, this surgery is performed on younger and younger patients, so the stent can be in the body for over 30 years. The stent cannot be removed from the patient, so the in vivo stability of the stent and the effects of the human organism on the stent can only be investigated on postmortem explanted stents. Therefore, this study deals with the non-destructive investigation of explanted coronary stents. Our goal was to determine the causes of visible damages and to measure the changes in the technical state.

Keywords: explanted coronary stent; non-destructive test; fracture; coating damage

\section{Introduction}

Over the last century, particularly in the last three decades, the occurrence of certain diseases in developed countries has increased significantly. These are known as civilization diseases, including a variety of cancers, cardiovascular diseases, hypertension and diabetes as well. These diseases occur mainly due to inappropriate lifestyle. The most important indicators are sedentary lifestyle, excessive stress, poor nutrition and a variety of addictions, e.g. alcohol and tobacco usage. Amongst these, ischemic heart disease (IHD) is the leading cause of death, accounting for around $11 \%$ of all deaths globally each year [1,2].

The effective treatment of IHD is angioplasty. During the procedure usually a so called stent is also implanted into the vessel. A vascular stent is a small slotted 
metal tube with a specific pattern. It is inserted into a vessel at the site of the narrowing (stenosis) to act as an internal scaffolding or support to the blood vessel and thus ensures the continuous flow of blood. Nowadays it is not uncommon that this surgery is performed on young and middle-aged patients, so the stent can be in the body for over 30 years [2].

Metal stents "last forever", they can't be removed from the body after implantation, therefore the investigation about the impact of the human organism to the stent has clinical relevance. Despite advances in polymer and drug technology, the metal alloy stent material remains a key determinant of clinical outcome [3].

During the life cycle of a stent, it has to withstand several mechanical loads. The stent is exposed to different kind of stresses during deployment, dilation and longterm usage. First the stent has to follow the vessel curvatures so it goes through a multiaxial bending. Due to the inflation of the balloon the stent has a uniform internal pressure and dilates. While expanding a stent we have to consider not only the tension effect of the balloon but also the impact of the plaque and the vessel curvature on the stent. In the body with every heart contraction the stent is exposed to the movement of the heart and the vessel. Assuming a 30 year long period, calculated with a heart rate of 60 beats per minute, nearly one billion contractions take place, so the stent bends the same number of times, which is a very high cycle fatigue load. The failure of the stent during deployment or usage (so during the useful life cycle) is clearly a negative impact on patients, so the discovery and the correction of any failures are very important $[4,5]$.

Most publications, which deal with stents after implantation, are from medical approach. Amongst these some pay attention on stent fractures. It has been already shown that the real occurrence of fractures are greater than reported in hospital records. One of the reasons can be the different interpretation of stent fracture (partial breakage or complete separation). Nakazawa et al. classified the fractures in five types; from one broken strut to the rupture of the stent. According to them, fractures occurred in $29 \%$ of all cases $[6,7]$.

There are only a few research papers dealing with explanted stents from the technical side. Halwani et al. investigated 9 excised vascular segment with 16 implanted stents. They found evidence of electrochemical and mechanicallyinduced corrosion on the stents. Vascular tissue surrounding corroded stents was shown to experience transfer of metallic elements [8]. These results, although coming from a limited number of specimens, encourage further investigation of the effect of in vivo corrosion on the structural integrity of stents.

This project covers the investigation of 13 coronary artery stents explanted from human cadavers (authorization number TUKEB 146/2012). All the explanted samples were subjected to several non-destructive tests (e.g. X-ray, CT) before the destructive ones. In our case non-destructive means that neither the deformations nor the material of the stents were damaged during the tests. Both bare metal 
stents (BMS) and drug-eluting stent (DES) were explanted. The stents were excised atraumatically with the vessels, they were implanted in, to avoid any damage to the stents. During the preparation of the stents the aim was to find the fastest method to remove the vessels from the stents without damaging them (or with the least amount of damage). $20 \mathrm{wt} . \%$ potassium hydroxide $(\mathrm{KOH})$ was used for 7-9 days on the vessels to complete the dissolution [9].

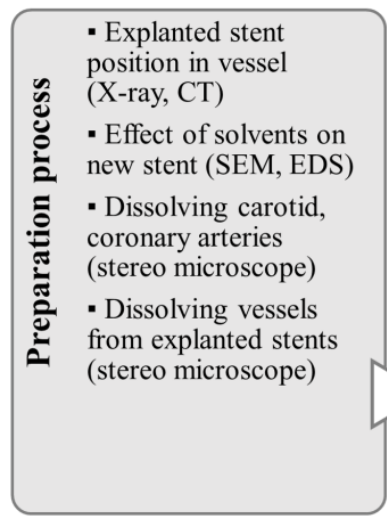

Investigation process of explanted coronary stents

\section{Figure 1}
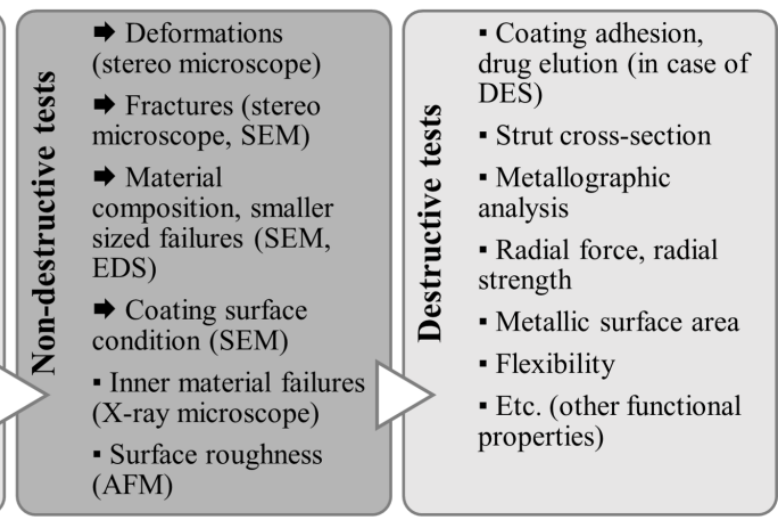

Coating adhesion drug elution (in case of DES)

Strut cross-section

Metallographic nalysis

Radial force, radial rength

Metallic surface area

Flexibility

Etc. (other functiona properties)

This paper covers the non-destructive investigation of the samples after the appropriate preparation process. There isn't any standardized process which is dealing with the examination of explanted stents so an investigation protocol was specified. The location of these tests in the protocol is shown in Figure 1. Our aim was to determine the outer deformations and failures of the explanted stents, scan the material composition, define the fracture surfaces and the coating surface condition.

\section{Materials and Methods}

\subsection{Preparation}

The 9 vessel samples with 13 coronary artery stents were explanted at the I. Department of Pathology and Experimental Cancer Research, Budapest, Hungary. The vessels were 3-4 times longer than the stents inside them, to avoid any damage to the stents during explantation. Before the experiments, X-ray and computed tomography (CT) images were taken of the explanted stents to see if we cause any damage of the stents. The images were taken from several directions parallel and perpendicular to the longitudinal axis of the stents with a Dage XD6600 X-ray (tube voltage $100 \mathrm{kV}$, tube power $0.90 \ldots 0.95 \mathrm{~W}$ ) and a GE Phoenix 
V $\backslash$ Tomelx M $300 \mathrm{CT}$. After the dissolution of the vessels from the stents, they were cleansed with ethanol to remove the solvent and any left vessel tissue. According to the input data, the X-ray images and the CT images, we could define the type of the explanted stents and give the main information about them.

\subsection{Materials}

More and more new stent materials appear including the promising bioabsorbable materials such as polylactic acid (PLA), but still physicians mostly use metal stents. Because of this and also because of the long-term follow-up of the previously implanted metal stents, our samples are made of the three most commonly used stent material; stainless steel (316LVM, X2CrNiMo18-14-3), cobalt-chromium alloy (L605, Co-Cr-W-Ni) and platinum-chromium alloyed steel (PtCr, Fe-Pt-Cr).

The summarized data about the investigated explanted stents that can be previously given is shown in Table 1 .

Table 1

Previous information about the explanted stent samples

\begin{tabular}{cccccc}
\hline No. & $\begin{array}{c}\text { Diameter* } \\
(\mathbf{m m})\end{array}$ & $\begin{array}{c}\text { Length* } \\
(\mathbf{m m})\end{array}$ & Material & Type & $\begin{array}{c}\text { Time spent } \\
\text { in the body }\end{array}$ \\
\hline$(1)$ & 3.50 & 12 & $\mathrm{PtCr}$ & BMS & 3 days \\
\hline$(2)$ & na & na & 316LVM SS & DES & 6 years \\
\hline$(3-1)$ & 3.00 & 20 & $\mathrm{PtCr}$ & BMS & 3 days \\
$(3-2)$ & 2.50 & 13 & L605 CoCr & BMS & 3 days \\
\hline$(4)$ & 2.50 & 20 & $\mathrm{PtCr}$ & DES & 2 years \\
\hline$(5)$ & 4.00 & 12 & $\mathrm{PtCr}$ & DES & 1 year \\
\hline$(6-1)$ & na & na & L605 CoCr & DES & 1 year \\
$(6-2)$ & na & na & L605 CoCr & DES & 1 year \\
$(6-3)$ & na & na & Na & BMS & 1 year \\
\hline$(7)$ & 3.00 & 24 & L605 CoCr & BMS & 3 days \\
\hline$(8-1)$ & 3.00 & 16 & L605 CoCr & BMS & na \\
$(8-2)$ & 2.75 & 19 & L605 CoCr & BMS & na \\
$(9)$ & 2.50 & 28 & L605 CoCr & BMS & 1 day \\
\hline$*$ size of stents according to manufacturer data &
\end{tabular}

\subsection{Methods}

Several recordings were made of the available coronary stents from different angles with an Olympus SZX16 stereomicroscope. We examined the fractures and the deformations of the stents. On the samples, where the geometry is not significantly damaged, nominal diameter and length values were also measured to indicate the recoil of the stents inside the vessel. In the case of broken or damaged 
stents the fracture surfaces were investigated with a Philips XL 30 scanning electron microscope (SEM). Images with both microscopes were also used to observe the quality of coating in the case of DES stents. EDAX Genesis and JEOL EX-54175JMU energy-dispersive X-ray spectroscopy (EDS) was used to determine the material composition of the samples (acc. volt. $15 \mathrm{keV}, 20 \mathrm{keV}$ ).

\section{Results}

\subsection{Stereomicroscopic Results}

First of all, every stereomicroscopic images were compared to the X-ray images to see if any deformation happened during the dissolution of the vessels. We can say that every deformation occurred before the preparation process. The stereomicroscopic images about the 13 stents explanted from the 9 vessels can be seen in Figure 2.

In the case of sample no. (1), the stent is deformed due to a serious, punctual stenosis. The stent was taken on the curve of the vessel and the plaque layer. There are no fractures, but the stent spent only few days in the patient, so we cannot speak about long-term impacts.

In the case of sample no. (2), the stent is seriously deformed and fractured, separated to two pieces, some struts are broken off. This stent spent the most time in the body ( 6 years). In addition to the fractures, the stent preserved its diameter.

In the case of samples no. (3-1) and (3-2), the stents are used for bifurcation stenting (T-stent technique). Physicians have to use this bifurcation stenting if the narrowing involves a main vessel and a side branch as well. During this method (3-1) stent was implanted in the main vessel so that the side branch ostium is approximately in the middle of the stent. After that a (3-2) stent is placed and dilated into the branch vessel through the expanded cell of (3-1) stent in the main vessel. The angle between the stents is $65^{\circ}$. The materials of the stents are different (Co-Cr and $\mathrm{Fe}-\mathrm{Pt}-\mathrm{Cr}$ ) which can be a problem with galvanic corrosion (electronegativity of the main elements: Co: 1.66, Cr: 1.88, Fe: 1.83, Pt: 2.28).

In the case of sample no. (4), a difference between the diameters (manufacturer's data and measured value) arises because the stent was dilated with 20 bar (instead of the 11 bar nominal pressure) according to the input data. Measurements also confirmed this.

In the case of sample no. (5), the stent is seriously deformed, which clearly shows that the stent has an open cell stent pattern. In this form the stent cannot properly carry out its function and in long-term the lumen of the vessel can decrease greatly. 


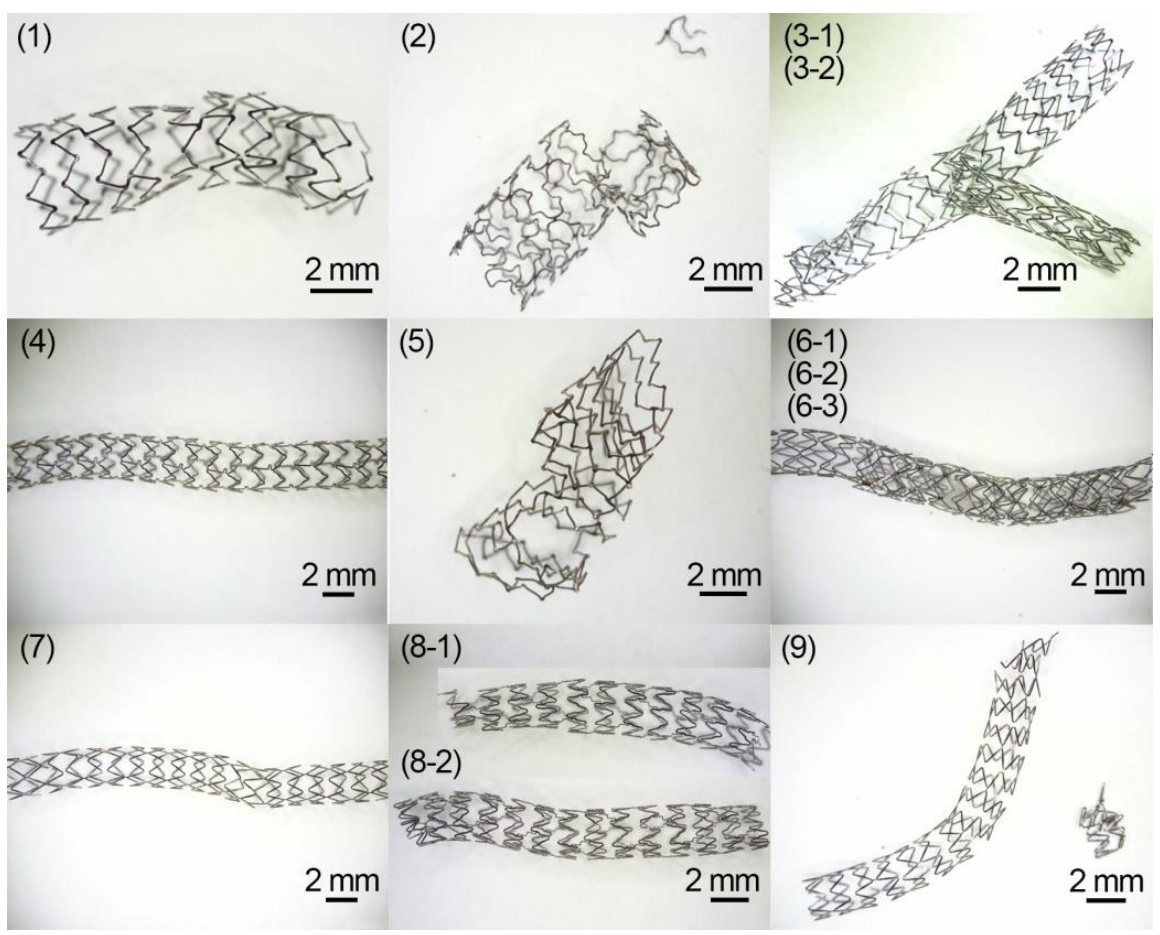

Figure 2

Stereomicroscope images about the explanted stents (with the numbered markings)

In the case of samples no. (6-1), (6-2) and (6-3), the three stents were overlapping in the vessel. (6-3) stent is over the other two stent and has markers to increase Xray visibility. One of (6-3) stent's strut is broken, presumably due to the overexpansion of (6-1) and (6-2) stent. This fracture does not hamper the stent in filling its function. Some parts of the plaque is still between the struts because the struts are too dense and it could not be removed without damaging the stents. It should take into account from the difference of the stent pattern between stent no. (6-1) and (6-3) that the materials are also different.

In the case of sample no. (7), the stent is only mild deformed, but the stent spent only few days in the patient, so we cannot speak about long-term impacts.

In the case of samples no. (8-1) and (8-2), according to the description the stents have to be overlapping, but the X-ray images clearly show that they were not, the stereomicroscope images also indicate this. In the case of stent no. (8-1) some struts at the end of the stent are broken, which can be occurred during the explantation procedure. The image clearly shows that the diameters of the stents are similar, although the nominal diameters are different. The diameter decreased along the entire length of both stents. On average diameter of stent no. (8-1) reduced with $15 \%$, diameter of stent no. (8-2) reduced with $10 \%$. 
In the case of sample no. (9), the stent is fractured to two separated parts and also seriously deformed at this part. As the stent spent a short time in the body, the fracture more likely the implication of a cutting during the explantation process. The broken struts are in a straight line which reinforces the idea that the stent was cut.

The summary of the stereomicroscope results can be seen in Table 2 .

Table 2

Summary about quality of deformation and fractures of the explanted stents

\begin{tabular}{|c|c|c|}
\hline No. & Deformation & Fractures \\
\hline$(1)$ & deformation in the middle, due to punctual plaque & No \\
\hline$(2)$ & serious deformation in the middle & $\begin{array}{l}\text { several struts, in the } \\
\text { middle }\end{array}$ \\
\hline$(3-1)$ & $\begin{array}{l}\text { serious deformation at one end, bifurcation stenting } \\
\text { (stent is in main vessel) }\end{array}$ & some struts, at the end \\
\hline$(3-2)$ & no deformation (stent is in side branch vessel) & no \\
\hline$(4)$ & no deformation & No \\
\hline$(5)$ & serious deformation & No \\
\hline$(6-1)$ & mild deformation, 3 stents overlapping & no \\
\hline$(6-2)$ & mild deformation & no \\
\hline$(6-3)$ & moderate deformation, over the other 2 stents & one strut, in the middle \\
\hline (7) & mild deformation & No \\
\hline$(8-1)$ & $\begin{array}{l}\text { mild deformation at the ends, } 2 \text { stents without } \\
\text { overlapping }\end{array}$ & some struts, at the end \\
\hline$(8-2)$ & mild deformation at the ends & no \\
\hline (9) & serious deformation at one end & some strut, at the end \\
\hline
\end{tabular}

\subsection{SEM Results}

Only the main results are shown according to SEM. The SEM images of stent no. (9) clearly show a cutting surface (Fig. 3 a-b). Based on the extensive regular planes and the grooves showing the edge of the cutting device, we can say that this damage occurred during the explantation process. In the case of stent no. (2) the SEM images show that two struts are facing each other (Fig. 3c). Fig. 3d shows the fracture surface of the stent no. (2). The classification of the surface is not clear. Ductile fracture can be ruled out, only brittle or fatigue fracture can be considered.

After the fracture of stent no. (2), the tip of the strut was exposed to abrasive impact, because the surface is smoothed on many places. The different crushes and small cracks on the side of the strut also indicates that the opposing struts were collided several times thus creating plastic deformation at the end of the strut. 


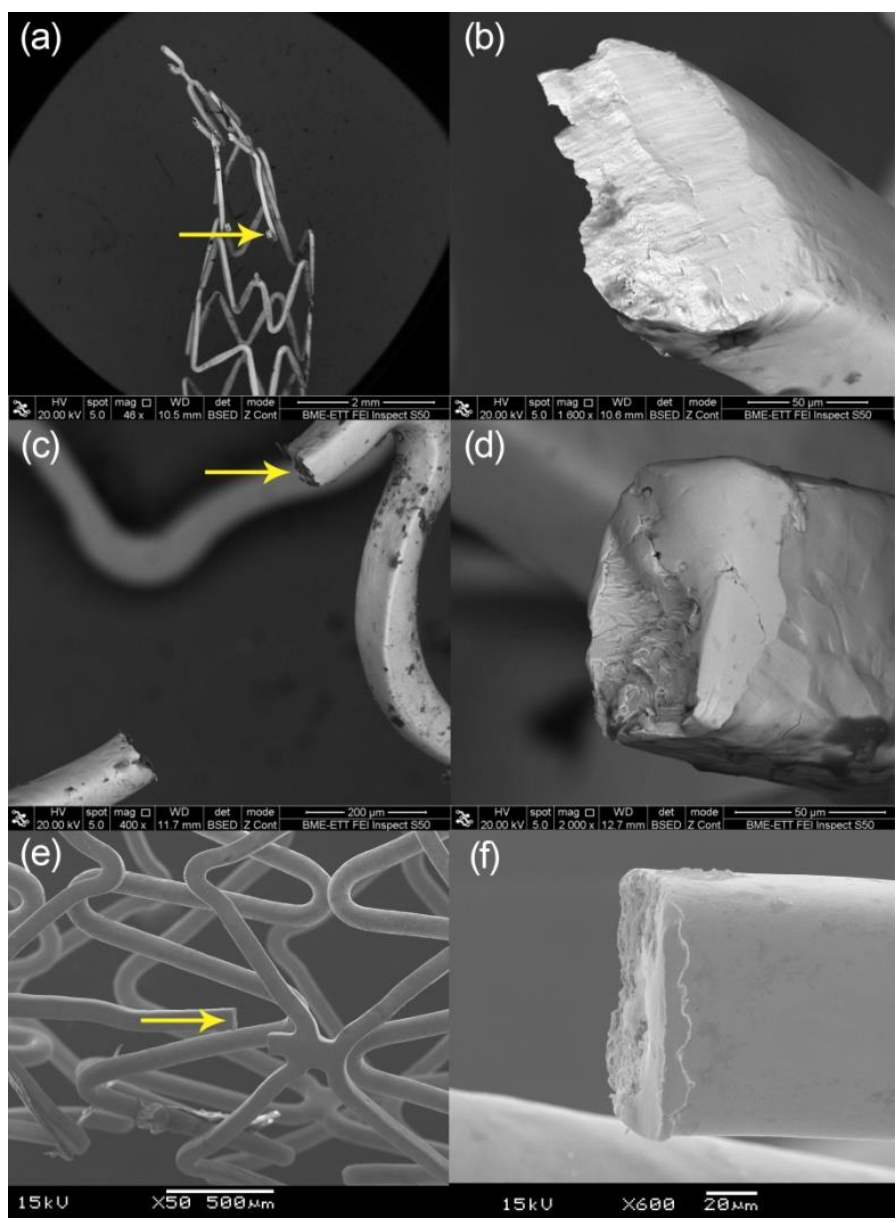

Figure 3

SEM image of a) stent no. (9) with marking the investigated strut, b) cut surface of (9) stent,

c) stent no. (2) with marking the investigated strut, d) fracture surface of (2) stent,

e) stent no. (6-3) overlapping on the other 2 stent, f) brittle fracture of (6-3) stent

Figure $3 \mathrm{e}$ and $3 \mathrm{f}$ show the fracture surface of stent no. (6-3), this is a brittle fracture most likely due to the over dilation.

After seeing the fractures of stents we thought it would be interesting to carry out a magnetic experiment. We used a simple permanent magnet to see if it attracts the stent or not, and the results show that most fractured stent are ferromagnetic. That is a huge problem if someone with an implanted stent needs radiology with magnetic resonance imaging (MRI), because the systems operate with a strong magnetic field (1-7 Tesla). The magnetic properties of fractured stents will be investigated with a vibrating sample magnetometer (VSM). 
According to the available SEM images we cannot drawn exact conclusions about the causes of the damages in the coating. Damages can be occurred during the crimping process, the expansion, the time spent in the body or the preparation process of the stents. On average the detachment of coating is started at the tip of the struts (Fig. 4). Although stent no. (5) was in the body for 1 year, other stents which were exposed to the body only for 1-3 days show also the same coating damages at the tip of the struts. It means that the coating damaged in the beginning of the treatment, and that is a very serious problem. The function of the coating is to form a border surface between the "foreign material" and the tissue to decrease the probability of in-stent restenosis and thrombosis. If it is damaged the metal surfaces further induce the inflammatory reactions.

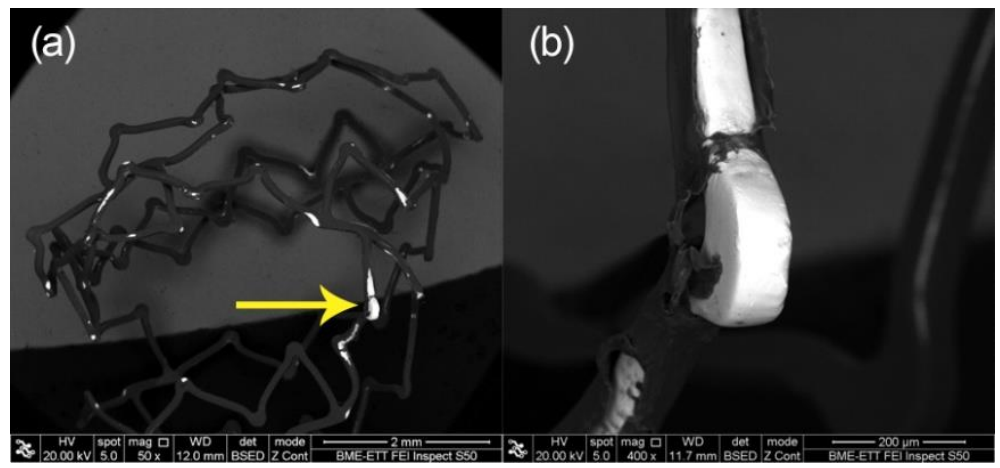

Figure 4

a) Coating of stent no. (5), b) detachment of coating at the tip of the strut

\subsection{EDS Results}

Our measurements show that there are no significant differences between the material standards (Table 3), the manufacturers' data and the measured material composition. The Fe-Pt-Cr alloy was developed by Boston Scientific, and currently only stents are made of this material, therefore there is no standardized composition for this material. We were interested especially in the quantity of $\mathrm{Ni}$ in the stent surface, because $\mathrm{Ni}$ is allergenic, but further investigation is needed to determine the aleatory $\mathrm{Ni}$ dissolution. In some cases we found a negligible amount of potassium, which is likely to be some residues remained from the vessel dissolution.

The material of stent no. (6-3) was unknown, we found that it is made of 316 LVM stainless steel, so our assumption is true that (6-3) stent is made of a different material than (6-1) and (6-2). The material of the markers is gold. Figure 5 shows the borderline of the marker and the base material, the assimilation of $\mathrm{Au}$ is satisfying and there aren't any signs of corrosion on the surface. 
Table 3

Summary about material composition according to the standards and the manufacturer data

\begin{tabular}{|c|c|c|c|}
\hline Alloy & ISO 5832-1 & $\begin{array}{c}\text { ISO 5832-5 } \\
\text { Wt. } \%\end{array}$ & $\mathrm{Fe}-\mathrm{Pt}-\mathrm{Cr} *$ \\
\hline $\mathrm{C}$ & $<0.03$ & - & $0.003-0.023$ \\
\hline $\mathrm{Si}$ & $<1.00$ & 0.40 & $<0.10$ \\
\hline Mn & $<2.00$ & $1.00-2.00$ & $<0.05$ \\
\hline $\mathrm{P}$ & $<0.025$ & 0.40 & $<0.01$ \\
\hline S & $<0.01$ & - & - \\
\hline $\mathrm{N}$ & $<0.100$ & - & $<0.01$ \\
\hline $\mathrm{Cr}$ & $17.00-19.00$ & $19.00-21.00$ & $17.50-18.50$ \\
\hline Mo & $2.25-3.00$ & - & $2.43-2.83$ \\
\hline $\mathrm{Ni}$ & $13.00-15.00$ & $9.00-11.00$ & $8.50-9.50$ \\
\hline $\mathrm{Cu}$ & $<0.50$ & - & $<0.02$ \\
\hline W & - & $14.00-16.00$ & - \\
\hline $\mathrm{Fe}$ & $\mathrm{x}$ & 3.00 & $\mathrm{x}$ \\
\hline $\mathrm{Pt}$ & - & - & $32.50-33.50$ \\
\hline $\mathrm{Co}$ & - & $\mathrm{X}$ & - \\
\hline
\end{tabular}

* manufacturer data

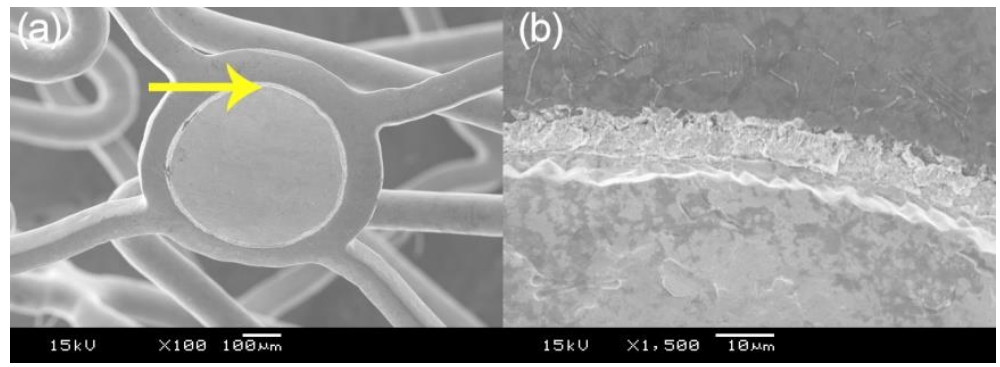

Figure 5

a) Gold marker on stent no. (6-3), b) borderline between the marker and the base material

Due to the position of the overlapping (6-1), (6-2), (6-3) and the bifurcation (3-1), (3-2) stents the examination of wear and corrosion can only be carried out by destructive tests (stent separation, maybe cutting).

\section{Conclusions}

During this series of experiments 13 explanted stents were investigated. Nondestructive test were performed; the outer deformations and failures of the stents were determined, the fracture surfaces were defined and the material composition was scanned. There is only limited information concerning the in vivo stability of explanted coronary artery stents, but still there are millions of metal stents implanted in people, therefore the investigation of explanted stents are well- 
established and important. Future lines of research will clearly be the continuation of the investigation protocol, beside this testing the formation time and causes of the coating damage and exploring the magnetic properties of stents is also justified.

\section{References}

[1] A. N. Nowbar, J. P. Howard, J. A. Finegold, P. Asaria, D. P. Francis, 2014 Global Geographic Analysis of Mortality from Ischaemic Heart Disease by Country, Age and Income: Statistics from World Health Organization and United Nations, Int. J. of Cardiol. 174:2 (2014) 293-298

[2] D. L. Mann, D. P. Zipes, P. Libby, R. O. Bonow, Braunwald's Heart Disease: A Textbook of Cardiovascular Medicine. Ch. 1 Fundamentals of Cardiovascular Disease, Saunders/Elsevier, 10 $0^{\text {th }}$ edition, Philadelphia, 2014

[3] E. Charpentier, A. Barna, L. Guillevin, J.-M. Juliard, Fully Bioresorbable Drug-Eluting Coronary Scaffolds: A Review. Arch. of Card. Dis. 108:6-7 (2015) 385-397

[4] R. V. Marrey, R. Burgermeister, R. B. Grishaber, R. O. Ritchie, Fatigue and Life Prediction for Cobalt-Chromium Stents: A Fracture Mechanics Analysis. Biomaterials 27 (2006) 1988-2000

[5] F. J. H. Gijsen, F. Migliavacca, S. Schievano et al, Simulation of Stent Deployment in a Realistic Human Coronary Artery. BioMed. Eng. OnLine 7:23 (2008)

[6] D. Alexopoulos, I. Xanthopoulou, Coronary Stent Fracture: How Frequent It Is? Does It Matter? Hellenic J Cardiol 52 (2011) 1-5

[7] G. Nakazawa, A.V. Finn, M. Vorpahl et al, Incidence and Predictors of Drug-Eluting Stent Fracture in Human Coronary Artery. J Am Coll Cardiol 54:21 (2009) 1924-1931

[8] D. O. Halwani, P. G. Anderson, B. C. Brott et al, Surface Characterization of Explanted Endovascular Stents: Evidence of in VIVO corrosion. J Biomed Mater Res B Appl Biomater 95B:1 (2010) 225-238

[9] D. Károly, D. Charalambous, B. Pogácsás, et al, Preparation of Explanted Coronary Stents for Investigation of Material Properties. Materials Today: Proceedings 3 (2016) 997-1002 